\title{
Políticas públicas de ciencia y tecnología, Y LOS RETOS ACTUALES DE LA EVALUACIÓN
}

\author{
Marta Cecilia Palacio Sierra ${ }^{1}$
}

\section{mesumen}

Este artículo plantea aspectos históricos y sociales acerca de los procesos de producción y evaluación de las políticas públicas de ciencia y tecnología. Se adentra en la crítica y el cuestionamiento a los enfoques teóricos tradicionales que median la acción política y propone el establecimiento de alternativas plurales, orientadas a ampliar la base de participación en la formulación y evaluación de las políticas públicas a partir de la búsqueda del consenso entre agentes sociales de múltiples procedencias.

\section{Absilact}

Historical and social aspects about production processes and technological and public science policies evaluation are posed in this work. Traditional theoretical approaches that mediate political action and propose the setting up of plural alternatives are deeply criticized and questioned. These are aimed to expand the participation basis of the proposal and evaluation of public policies, starting from the agreement among social agents from different origins.

1 Administradora de Empresas Agropecuarias de la Corporación Universitaria Lasallista. Socióloga de la Universidad Pontificia Bolivariana, Magíster en Sociología de la Educación de la Universidad de Antioquia. Candidata al doctorado en Estudios de Ciencia y Tecnología de la Universidad del País Vasco. Asesora de Línea de Investigación del Instituto Tecnológico Metropolitano.

(marthapalacios@itm.edu.co). 


\section{Palabras clave}

Políticas públicas, Ciencia, Tecnología, Innovación, Evaluación, Pluralismo.

\section{Key words}

Public politics, Science, Technology, Innovation, Evaluation, Pluralism. 


\section{UNTRODUCCIÓN}

Las políticas públicas que orientan el deber ser del desarrollo de la ciencia, la tecnología y la innovación en un país, aparecen como el producto de un consenso que refleja las decisiones tomadas por las agencias del Estado, a partir de criterios implícitos, en donde unos agentes sociales se hacen responsables de definir el rumbo de la investigación científica y tecnológica, a través de políticas, cuyos resultados se objetivan y expresan en la determinación de prioridades frente a las líneas de investigación, la asignación de presupuestos en sus diversas cuantías, la selección, aprobación o rechazo a los proyectos presentados a través de las convocatorias públicas, dirigidas a los grupos y centros de investigación, independientes o adscritos a las instituciones educativas de nivel superior.

Las políticas públicas ${ }^{2}$, en materia de ciencia y tecnología, logran trascender procesos sociales complejos de "desacuerdo teórico", de conflicto de intereses, "de conjunción de valores epistémicos y no epistémicos" y se constituye en un eje rector que genera lineamientos de apariencia neutral, frente a los cuales deberán converger los diversos agentes sociales comprometidos con la producción, distribución, uso y apropiación de la ciencia y la tecnología en un país o en una región.

Indagar acerca de los puntos de confluencia entre las acciones de los agentes estatales, encargados de la producción de políticas públicas, los métodos y prácticas concernientes a la evaluación, el cambio de estrategias y los procedimientos utilizados para el rechazo, aceptación, valoración o evaluación de la actividad científica

2 Según Omar Guerrero el concepto referido a la "voz pública, igual que el vocablo política, tienen la misma raíz etimológica a histórica: ambas palabras derivan de la raíz pul (multiplicidad, amplitud); de ella derivó en el griego la palabra polis (ciudad, Estado), origen del concepto de política y, en el latín, populus (pueblo), que es un sustantivo producido por la reduplicación de la voz pul". Así política y pública, poseen un parentesco etimológico y un significado común ya que hacen referencia a lo colectivo y comunitario (Guerrero, 1997: en la red). 
y tecnológica, puede ser un soporte útil para la comprensión de los procesos que orientan la toma de decisiones con miras a lograr el avance de la ciencia y tecnología, especialmente en los países con menos desarrollo.

A su vez, representa una posibilidad para la generación de alternativas analíticas que propicien la apertura de espacios de aprendizaje, con miras a cualificar las acciones requeridas para el estudio y evaluación de dichas políticas.

Por ello, en este artículo, se realiza, en un primer momento, una breve indagación acerca del contexto relativo al accionar de las políticas públicas ${ }^{3}$ y por extensión, a las que están referidas a la ciencia y la tecnología; de manera específica se abordan algunos aspectos acerca del Estado y los procesos de decisión, como categorías que se encuentran presentes en los estudios actuales sobre la materia.

En una segunda instancia, se hace referencia a los componentes teóricos y conceptuales resultantes de los análisis históricos que se han realizado frente a las formas que asumen los procesos de evaluación. Se trata de hacer una delimitación en el campo de estudio de las prácticas de agentes, cuya actividad versa sobre la generación, evaluación y cambio en materia de políticas públicas y su incidencia en la interacción de los diversos agentes sociales, en el ámbito nacional, regional y local.

Como resultado de las discusiones presentadas, se resalta la necesidad del encuentro de marcos comunes de actuación en el ámbito nacional y regional, con miras al logro de una cualificación de las agendas políticas, buscando con ello que se potencien las acciones para el logro de acuerdos mínimos y la generación de espacios de discusión compartidos, como alternativa orientada a la democratización de la ciencia y la tecnología, lo que implica, buscar equidad en el acceso al conocimiento, su producción, uso y distribución.

3 Según Maldonado (2005), sólo puede hablarse de políticas públicas con referencia al Estado y explica que en instancias tales como departamentos y municipalidades no existen políticas públicas en el sentido político, sino en el sentido administrativo. 


\section{POLITICAS PUibLCAS UNA CONCEPTUALIZACIÓN NECESARIA}

Lo político, junto con lo ético y lo estético, hace parte de los grandes ejes articuladores de la cultura de la humanidad, dada su incidencia en todas y cada una de las formas socioculturales de que se tenga conocimiento. Por ello, la preocupación por las acciones públicas, enmarcadas bajo el concepto más restringido de lo político, han caracterizado las diversas reflexiones que desde la antigüedad hasta hoy, han hecho no sólo los encargados del gobierno, sino también los filósofos, científicos y ciudadanos del común; donde éstos participan, en mayor o menor medida, en la discusión frente a su impacto social, pero no así, en su formulación, evaluación y cambio.

Los enfoques pluralistas se presentan por el contrario, como una alternativa para las políticas públicas, ya que asumen el reto de centrar el interés en las interacciones de los agentes sociales, su entorno, intencionalidades y mediaciones en el uso de dispositivos de carácter instrumental y en especial, hacen hincapié, en las finalidades de las agencias que son portadoras del encargo social de su producción, ejecución y evaluación, en ámbitos concretos, delimitados por territorios específicos.

El pluralismo de dichos enfoques responde en gran medida a una necesidad social, surgida ante la disminución de efectividad de las grandes narrativas de la modernidad, que han perdido su vigencia estratégica ante el redimensionamiento de lo global y lo regional, como focos de interés para la acción política.

Lograr un acercamiento comprensivo a la denominación genérica del concepto políticas públicas, implica delimitar su campo de acción a aquello que hace referencia a la definición de propósitos con un alcance temporal, legítimamente establecido a través de propuestas programáticas, provenientes de las autoridades que poseen la competencia para su establecimiento en un determinado territorio.

Esta delimitación requiere además, la separación funcional frente a acepciones que incluyan en el concepto de políticas públi- 
cas, las acciones relativas a la disputa por el establecimiento de controles efectivos del poder o por lo que tradicionalmente se ha conocido como el gobierno de sociedades y grupos comunitarios. (Cozzens y Woodhouse 1995: 540).

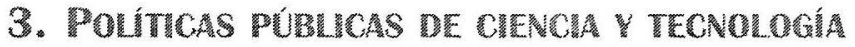

El surgimiento de las políticas públicas como campo de conocimiento, tiene lugar en los Estados Unidos poco después de la Segunda Guerra Mundial y se extiende rápidamente a Europa, con el interés creciente de consolidar la reflexión sistemática, frente a las tareas que ha de realizar el "estado benefactor". En este sentido también las políticas para incentivar el desarrollo de la ciencia y la tecnología, tuvieron su origen en esa época, especialmente, a través del fomento a la investigación básica, conforme al modelo propuesto en los estudios realizados por Vannevar Bush en 1945.

Si bien estas políticas cobran un claro énfasis en pro del desarrollo científico y tecnológico en las regiones más desarrolladas, en América Latina y el Caribe, toman su expresión bajo la forma de transferencia tecnológica. Es de resaltar que la CEPAL ${ }^{4}$ jugó un papel fundamental en la generación de una postura reflexiva, frente a lo que pueden ser los primeros estudios que en la región, contribuyeron a problematizar, desde el punto de vista económico, social y político, la relación entre la ciencia, la tecnología y el desarrollo.

Las tendencias adoptadas por la CEPAL en materia de políticas publicas de ciencia y tecnología plantearon una relativa independencia respecto a los centros de poder mundial (Albornoz, 2001: 4), e incluso a sus modelos, proponiendo formas alternativas para construir lo que en su momento denominaron "ciencia propia",

4 La CEPAL tuvo su origen en las preocupaciones por la economía latinoamericana. Se impulsaron desde allí las políticas de industrialización basadas en la sustitución de importaciones, donde el Estado juega un papel preponderante para efectos de la regulación del mexcado y se exploró por primera vez en la región, la formulación de políticas para el fomento de la ciencia y la tecnología. 
actitud crítica que se acompañó de la formulación de modelos de desarrollo, que si bien reconocían el significativo aporte de la ciencia y la tecnología del momento, reclamaban la formulación de políticas públicas que consultaran el contexto específico de su producción.

Por ello, en esa época se pudo observar la dualidad del impacto de los dos modelos que coexistieron, hasta el momento de la irrupción de los procesos de globalización de la economía, uno, centrado en la apropiación y transferencia en la producción de ciencia y tecnología, destinada en lo fundamental a la sustitución de importaciones en el sector industrial, y otro, que fomentaba la producción de ciencia y tecnología, con mayor arraigo en el sector agrario, sentando las bases para el gran auge en la creación de centros de investigación, al servicio de las necesidades de los productores del sector rural.

En la mayoría de los países de América Latina y el Caribe, las políticas de ciencia y tecnología fueron el fundamento para la consolidación de la investigación en campos como el de la generación de energías alternativas, desarrollo de variedades propias para los cultivos, según la vocación agrícola de las regiones, la incursión en el área de la biotecnología, la recuperación y difusión de las prácticas agropecuarias tradicionales como alternativas de producción limpia, el control biológico de plagas, entre otros; investigaciones que marcaron un hito en cuanto a la vinculación de científicos, tecnólogos, grandes, pequeños y medianos productores, agentes del gobierno y sector educativo formal y no formal, todos ellos trabajando en pro de la producción, distribución y uso de la ciencia y la tecnología, bajo condiciones de institucionalidad, financiación y participación, únicas en la historia de la región.

Dichas prácticas fueron desmontadas paulatinamente, para dar paso a aquellas que se guían por enfoques neoliberales donde prima el espíritu de privatización, la búsqueda de la eficiencia y la eficacia en la producción de la ciencia y la tecnología, hechas a través de institutos, centros de investigación y universidades, con un alto grado de dispersión y desintegración, con respecto a 
los sectores sociales y productivos, con una gran disminución de la inversión pública en investigación, pero con incremento de los costos de operación y funcionamiento de los organismos encargados del control del gasto público. (Jaramillo y Chaparro, 2004).

Al decir de Roth (2004: 16), en los países de esta región, el foco del análisis en materia de políticas públicas en los años 60 y 70 , estuvo centrado en las decisiones atinentes al logro del bienestar y la realización de estudios comparativos que contribuyeran a la superación de los problemas del desarrollo, para luego desplazarse hacia la búsqueda de los componentes de constitución de agendas gubernamentales, y luego avanzar hacia la comprensión y promoción de las comunidades, sistemas y redes, responsables del accionar de las políticas públicas.

La evaluación y la gestión de dichas políticas y sus especificidades, como es el caso de las políticas ambientales, en ciencia y tecnología, en salud y educación entre otras, han centrado sus búsquedas en la eficiencia de la gestión pública, enfoque que sintetiza la estrategia básica para el logro de las aspiraciones en los estados neoliberales del actual contexto político internacional.

En los años noventa y principios del presente siglo, dos componentes de naturaleza diversa pero indisociable, han marcado el centro de interés en las prácticas de las políticas públicas:

- La búsqueda de la democratización en la acción del Estado, mediada por procesos de participación y construcción de autonomía en los contextos regionales y locales, que se expresa en la generación de procesos crecientes de descentralización político administrativa y en la introducción de agendas políticas con medidas para atender los problemas crecientes de pobreza, terrorismo, violencia, narcotráfico, inseguridad, corrupción, pérdida de competitividad de las empresas, entre otros.

- Y la elaboración de agendas políticas que tratan de subsanar el cada vez más notorio desajuste entre el avance tecnocientífico, en la esfera internacional y la carencia de procesos permanentes de producción, distribución y uso apropiado de la ciencia y la tecnología nacional, que no logran orientarse de 
manera adecuada a dar respuesta a las múltiples demandas de la región.

La inclusión explícita y programática de todas las temáticas señaladas, en las distintas agendas políticas en los países de la región, durante los últimos 20 años, no necesariamente ha contribuido de manera significativa a enfrentar las demandas sociales, ya que su configuración obedece a una lógica fragmentada por los períodos de gobierno, que se traduce en programas de acción, derivados de procesos de decisión, cuyas aspiraciones no trascienden la búsqueda de realizaciones a nombre de agentes particulares, sin llegar a convertirse en políticas de Estado, lo que restringe las posibilidades de impactos permanentes, de amplia cobertura, y que partan de la consulta a las demandas sociales y cuenten con una participación ciudadana autónoma y racional (Olivé: 2003).

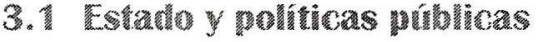

Dado que el aparato estatal no tiene el alcance necesario para penetrar, en toda su magnitud, los diversos espacios sociales, se enfrenta a innumerables fuerzas de resistencia, que se constituyen en nodos o centros a través de los cuales también se realiza el ejercicio del poder, poniendo en evidencia la dificultad para ejecutar sus políticas, esto le da un carácter de ilegitimidad frente a algunas de las regulaciones que pretende aplicar en la sociedad.

Recurre por lo tanto a la adopción de reglamentaciones jurídicas para ganar legitimidad en sus estrategias. Es éste el resultado de la movilización de poderes públicos y privados, portadores de intereses contradictorios que disputan su asiento en las políticas públicas, a manera de escenario donde logran su expresión las múltiples interacciones que establece el Estado con todos y cada uno de los agentes sociales.

Las políticas públicas se convierten así en la representación del complejo entrecruzamiento de los diversos sistemas de acción, que operan en un determinado territorio, orientados por flujos de información y que tienen como objeto común la acción pública. Cabe 
anotar que más allá de acciones gubernamentales, son actuaciones de seres humanos que toman decisiones acerca de su desarrollo e implementación y realizan los procesos de evaluación que determinan su modificación o permanencia.

Si bien los contornos de una política son dificiles de demarcar, su estudio exige delimitaciones en las cuales, éstas sean asumidas como constructos sociales de instituciones públicas, de sus agentes, valoraciones y sistemas de elección y decisión que se ponen en juego en interacciones complejas.

Los agentes que las dinamizan han sido tradicionalmente, un limitado número de personas que toman decisiones y donde se revela la configuración de la repartición del poder en el seno del Estado. Por ello, es posible llegar a percibir a través del análisis de los procesos de generación de las políticas públicas, los entramados de legitimación y los procedimientos que asume un determinado Estado, en la búsqueda de su permanencia.

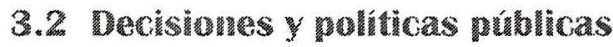

La orientación en la definición de una política, se da a partir de decisiones que se derivan de elecciones entre una pluralidad de alternativas, que tienen por objeto presentar soluciones, proponer metas, medios, metodologías y objetivos para resolver problemas. En este tipo de decisiones confluyen intereses y recursos de diversa índole, provenientes de agentes y agencias tanto públicas como privadas, que no necesariamente obedecen a procesos similares de toma de decisiones y que incluso, rechazan los esquemas difundidos en la actualidad caracterizados por su aspiración al logro de la racionalidad por la vía de la búsqueda de la eficiencia.

El modelo taylorista para la toma de decisiones, también denominado tradicional, se plantea como un proceso mediante el cual se busca lograr consensos frente a una determinada alternativa, a través de la discusión entre los miembros del grupo decisor, y que puede verse entorpecido por la carencia de habilidades técnicas o adecuadas relaciones interpersonales, por parte del agente que 
asume el carácter de líder en el grupo que debe tomar la decisión. (Certo S. 2001: 147).

En las sociedades contemporáneas se ha abierto otra vía en la toma de decisiones, la tecnocrática, que remite a la definición de los presupuestos requeridos para elegir entre una u otra alternativa, a partir de la vinculación de la ciencia y la tecnología como saberes necesarios a la hora de tomar decisiones.

Estas actividades se hacen cada vez más complejas, ya que las tensiones que se crean entre saberes políticos, saberes expertos y democracia, no siempre son puntos de encuentro para el ejercicio de la responsabilidad social en la toma de decisiones, situación que ha favorecido la vía de la tecnocracia como único camino para salvaguardar a los agentes gubernamentales de las responsabilidades en la elección en materia de políticas públicas. Queda por tanto abierta la pregunta acerca del ¿cómo lograr interacciones entre los agentes estatales políticos, científicos y tecnólogos que conduzcan a la obtención de realizaciones en el marco de la democracia?

En el modelo tecnocrático, como lo expresa Habermas (2001: 134), el Estado abandona sus prácticas coactivas basadas en intereses, para convertirse en "órgano ejecutor de una administración integralmente racional", esto es, se subordina el político al científico. Habermas hace una crítica a estos dos modelos y propone un tercero, el pragmático, a través del cual se hace más factible el acceso a la democracia, mediante la inclusión del público en un diálogo en el cual éste exprese abiertamente sus opiniones y se logre conciliar el saber técnico con las valoraciones provenientes de los políticos, para generar amplios procesos de aprendizaje, donde haga presencia el diálogo de saberes y la confrontación entre colectivos.

Este modelo está orientado a la búsqueda de la participación de los diversos agentes sociales, participación que resulta aun más compleja de poner en práctica, cuando se trata de la ejecución de las políticas públicas, bajo los presupuestos emanados de agencias cuyo interés fundamental es el logro de la eficiencia. Cabe entonces preguntarse de nuevo ¿cuál es la compatibilidad de "la eficiencia a toda costa" con la democracia? 


\section{Evaluación de políticas púbilicas}

La evaluación es en la actualidad una práctica que tiene como finalidad la producción del cambio total o parcial, el ajuste o modificación de las políticas públicas; se encuentra emparentada con los progresivos cuestionamientos frente a las acciones del Estado y su consiguiente pérdida de legitimidad.

La proliferación de críticas a lo largo del periodo de la posguerra y hasta muy avanzada la década del sesenta, se centró en lo concerniente al manejo de las políticas sociales y muy especialmente, frente a las que han orientado el sector de la economía, cuestionando en general su actuación como Estado benefactor.

A partir de estos años, los países de Europa occidental y los Estados Unidos comenzaron a transformar los métodos de la administración pública, dándole un viraje hacia nuevas formas y procedimientos, que sustituyeran la dificil tarea de lograr objetivos de desarrollo a través de políticas intervencionistas, tratando de contrarrestar con eficiencia y eficacia, la cada vez más costosa labor del Estado.

Este cambio de perspectiva se tradujo en un acercamiento a los enfoques de la gestión empresarial del sector privado, en el cual se habían incorporado, de tiempo atrás, la "teoría de la decisión racional" y los modelos de planeación estratégica; los cuales tienen en común, la práctica de fijación de fines a ser logrados a largo plazo. Este tipo de racionalidad presenta limitantes en el sentido de que no permite abrir la discusión acerca de los fines, como dice Olivé, [2000: 153] para el caso de la elección racional de las teorías científicas, se da gran importancia a la elección de los medios que propician el logro de los fines, pero no se considera pertinente la pregunta acerca de si los fines que se persiguen son adecuados.

El proceso que se desprende de los enfoques utilizados para la gestión de las políticas públicas, parte de la definición de los fines, sin reconocer que esta acción no está exenta de una orientación valorativa, y es en este espacio -el de los valores-desde donde es posible ser crítico, "justificar los fines, y no al revés". Entendiendo 
además, que no hay unos fines últimos y definitivos, sino más bien una pluralidad de fines, como lo expresa Echeverría (2002: 116). Este presupuesto, si bien el autor lo contempla útil por su contribución al análisis de la ciencia y la tecnología, también permite orientar el análisis de las políticas públicas.

Los permanentes cuestionamientos a este tipo de países, provenientes de los organismos internacionales, en el contexto externo, y de los diversos sectores sociales en el contexto interno, han hecho que la búsqueda de legitimidad asuma dos formas de proponer e implementar estrategias para la acción pública; en ambas se expresa la tensión entre la concepción tradicional de la legitimidad, basada en la imposición de la norma y aquella que se sustenta en el logro de los objetivos y metas propuestas en los planes y programas resultantes de la aplicación de las políticas de Estado. Se trata de oponer la ley, a la manera de las prácticas tradicionales, al logro de objetivos, esto con el ánimo de ganar en eficacia y eficiencia en la gestión pública, abriendo un nuevo rumbo en materia de evaluación.

Esto ha conducido a que los enfoques en la evaluación, que predominaron entre los gestores de la actividad pública, se transformen poco a poco, para proveer al Estado, a los gobernantes y en especial a los ciudadanos, de la información requerida para sopesar las consecuencias desencadenadas en el presente y a futuro, por las decisiones y acciones, de las cuales debe hacerse responsable el gobernante.

Es en este contexto donde se ubican las prácticas evaluativas en la acción gubernamental, concebidas tradicionalmente como la determinación del grado de eficiencia y eficacia de las políticas públicas y sus efectos a nivel de logros y fracasos ocasionados por la intervención en determinadas áreas problemáticas de la sociedad.

Las políticas públicas presentan distintos niveles de complejidad, de acuerdo a las áreas que intervienen, esto lleva a que la especificación de criterios que se pueden asumir como indicadores para las evaluaciones, tengan grandes diferencias. Las políticas que presentan metas y objetivos definidos en torno a una problemática, 
presupuestos claros, demandas específicas de sus destinatarios, delimitaciones de carácter temporal, tienden a hacer de estos componentes, los rasgos principales de sus protocolos de evaluación, estos son rasgos muy comunes en las políticas de ciencia y tecnología, y las relacionadas con el medio ambiente.

Este tipo de políticas plantean retos que, en regiones como Latinoamérica, no se asumen a través de la generación de estrategias de evaluación pertinentes, que permitan superar las tradicionales prácticas de traslado de indicadores, de una nación considerada desarrollada, a otra que no lo es (Ibarra, A.: 2006). Esto hace que dichos modelos se implementen con ninguna o una mínima adaptación, desconociendo las demandas de los diversos agentes sociales al estructurar los sistemas de evaluación.

En este sentido, es valioso el modelo que presenta J. Echeverría (2001: 139), para el análisis axiológico de la acción tecnocientífica, entendida como un sistema complejo de acciones que no pueden ser analizadas aisladamente, sino por el contrario, deben ser consideradas en el marco de un contexto situado temporalmente y en sus múltiples interacciones, donde cada componentes debe ser evaluado a partir de un sistema de valores, que incluye un conjunto de subsistemas imbricados en una determinada estructura, en la cual Echeverría resalta los siguientes componentes: los agentes, el hacer, el objeto, los instrumentos, escenarios, condiciones sociopolíticas, finalidades, resultados y consecuencias.

Estos componentes puede ser abordados en una evaluación de manera conjunta o separada, teniendo presente que no se aplican los mismos sistemas de valores. Sin embargo, al momento de realizar la reconstrucción de todo el proceso de evaluación, se deben tener en cuenta de manera conjunta los criterios de valoración aplicados y sus diversas interrelaciones.

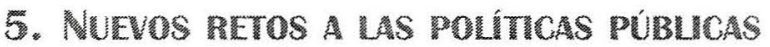

Asumir las políticas públicas desde estas nuevas perspectivas que han sido desarrolladas para la evaluación de las acciones tecnocientíficas, puede conducir a superar la sumisión a la nor- 
matividad del poder político, a través de la inclusión de una deliberación amplia, contextualizada y con la medición de indicadores autorreferenciados.

A pesar de la gran variedad de enfoques que se aplican en los procesos de evaluación, en los sectores vinculados con la actividad gubernamental, se tienden a asimilar como función evaluadora, a todas las actividades que ejercen control sobre los distintos agentes que a ellas se vinculan, agentes gubernamentales, contratistas 0 personal independiente encargado de la ejecución de las políticas, se incluye en esta función la vigilancia y la generación de sanciones, hasta llegar al punto de convertir dichas prácticas, en un elemento más del ejercicio del poder en su componente represivo.

Se propician a partir de allí, una serie de actitudes de rechazo frente a la evaluación, que conducen a la aceptación por imposición, a la resistencia pasiva, al ocultamiento por temor a la sanción y toda una serie de actitudes que desvirtúan los procesos de evaluación. De otro lado, está el desarrollo de la función pública como el ejercicio de la burocracia, donde funcionarios que en el cumplimiento de las reglamentaciones, realizan análisis de las acciones desde una sola perspectiva, la que proviene del reglamento, lo que le da a la evaluación un carácter eminentemente normativo.

En la gestión pública contemporánea, la evaluación se desarrolla desde sus inicios, a la manera de la instrumentalización de procesos que asumen como objetivo la búsqueda de la racionalidad en las elecciones de opciones técnicas; allí juegan un papel fundamental los expertos externos como "garantes de neutralidad". Pero como se ha dicho, en los procesos de evaluación confluyen los intereses de distintos agentes, y por lo tanto es un contrasentido aspirar a que los evaluadores hagan uso de una pretendida "neutralidad valorativa", o que actúen desde la perspectiva de los agentes solicitantes de la evaluación, esto es, que asuman sus valores y percepciones e interpreten desde allí la normatividad.

Esta situación plantea dos tareas relevantes para el experto, una de ellas es determinar la validez de las evaluaciones y su capacidad para delimitar los verdaderos efectos de una política, lo que 
significa establecer si existen nexos causales entre una aplicación y sus posibles consecuencias.

Otra tarea relevante para los expertos, es la elección y aplicación de unas estrategias y herramientas metodológicas que garanticen la calidad del procedimiento utilizado para la realización de las evaluaciones. Ambas tareas cobran una gran relevancia, dado el estatus tan valioso que han adquirido las prácticas evaluativas como instrumento básico para lograr la impronta de eficiencia que requieren los procesos de conocimiento y mejoramiento de las acciones públicas.

Las principales críticas en materia de evaluación se refieren a la notoria carga positivista que contienen y que inducen a considerar como válida una sola metodología, una sola definición e intencionalidad de la evaluación y una única acepción de racionalidad; valga la claridad que al respecto hace Olivé citando a Mosterín [2000: 152], la racionalidad no es una facultad, sino un método. Pero además, la racionalidad no es única, en las sociedades democráticas se debería hablar de diversos métodos racionales que contribuyan a la elección de alternativas.

La búsqueda de cualificación de la democracia en las sociedades contemporáneas, hace inminente la introducción de la complejidad y la exclusión de perspectivas lineales, en los proceso de elaboración de planes y programas para la ejecución de las políticas públicas; la aceptación de la complejidad introduce necesariamente la consideración del pluralismo de los valores presentes en todas y cada una de las acciones públicas.

Realizar una evaluación bajo estos presupuestos, implica aceptar que el juicio de los expertos no está exento del juicio de valor, y que por lo tanto, si se busca la ampliación de las bases democráticas de la sociedad, se requiere de evaluaciones que sean portadoras de la diversidad de valores que hacen presencia en la sociedad.

Aunque de allí, no se desprende que se esté en la vía para lograr evaluaciones aceptadas por consenso, la dinámica generada en espacios de carácter científico, tecnológico, empresarial, y en especial en proyectos de gran magnitud, como es el caso de los relacionados 
con la problemática ambiental, obligan a plantear el requerimiento de perspectivas transdisciplinarias; las cuales permiten comprender que la evaluación es una actividad que debe acompañarse de dimensiones cognitivas e interpretativas, complementarias a las ya tradicionales dimensiones, que proveen de los instrumentos técnicos y la normatividad existente en un determinado contexto social, en donde la apertura a la negociación entre agentes sociales provenientes de diversos sectores, implica tener en cuenta que la percepción, los intereses y la postura de "expertos" y "no expertos", se expresan en los procesos de evaluación mediante la reflexión y el aprendizaje colectivo.

\section{Concluslones}

A manera de conclusión puede decirse que las evaluaciones con enfoques alternativos -a los enfoques tradicionales donde prima la visión del agente interno, perteneciente a las entidades gubernamentales o la visión del agente externo, científico o técnico-parten del presupuesto de que la evaluación de una política pública está en relación directa con el evaluador y su posición en el contexto de lo evaluado, careciendo de similitud los criterios del político a los del científico, del usuario y demás participantes de un proceso evaluativo. Se reconoce, por tanto, que cada uno de los agentes sociales es portador de intereses diversos y enfoques cuyas metodologías de acercamiento a los fenómenos sociales, contribuyen a visibilizar el mundo de manera diversa.

Estos enfoques posibilitan el establecimiento de alternativas plurales para la evaluación, ofrecen a su vez, criterios flexibles y metodologías cualitativas orientadas a ampliar la base de participación entre agentes de múltiples procedencias y a abren espacios que propician el diálogo entre los diversos agentes sociales políticos, expertos y ciudadanos, buscando consensos y negociaciones entre ellos

Además, desarrollan estrategias que se insertan a la actividad política, a través de la construcción de comunidades de discusión y aprendizaje colectivo, cuyos objetivos ya no radican en encontrar 
la mejor solución o la más eficiente, sino la que pueda satisfacer más ampliamente y según el contexto, a los agentes sociales que aspiran ver colmadas sus expectativas, a partir de los logros de programas originados en políticas públicas, que se asumen como experiencias que contribuyen a la transformación individual y la de los sujetos colectivos.

En este tipo de propuestas, cabe esperar que la búsqueda de consensos en la definición de políticas públicas, no se opere por la dominación de un grupo de agentes sociales sobre otro, sino que los procesos de decisión, evaluación y cambio de acciones, que a la manera de las comunidades científicas, se orienten hacia el logro de acuerdos significativos, a través del "escrutinio y el debate comunitarios" (Pérez Ransanz 1999: 173), donde los estándares de evaluación sean flexibles, compartidos, y a su vez, sujetos de evaluación, a partir de la especificación de los fines que se persiguen y con arreglo a los cuales se han elegido.

Esto es, asumir la búsqueda de los consensos por la vía racional de la deliberación, para lo cual se hace necesario aprender de las comunidades científicas y alejarse de las prácticas de manipulación y el engaño, que se imponen a través de la fuerza o la moda, buscando con ello, y en palabras de Kuhn, "acuerdos entre expertos, cuyos juicios se basen en el dominio de los criterios, los procedimientos y la información pertinente, sin que se aspire a través de estos juicios, al logro de elecciones de validez absoluta" (citado por Pérez R., 1999:150).

Por ello, cada vez se hace más necesario que la acción política se acompañe de un gran esfuerzo por parte de los agentes sociales (Olivé, 2000:137), para dar un salto hacia el pluralismo, tratando de apartar las políticas públicas de ideas acerca de la existencia de fines, métodos y valores únicos. 


\section{Biblografia}

ALBORNOZ, Mario: 2003. Elogio de la perplejidad, elogio de la incertidumbre y elogio de la voluntad. En: ENCUENTRO INTERNACIONAL CAMBIO TECNOLÓGICO, INNOVACIÓN Y DESARROLLO: La ciencia y el destino de los pobres. Medellín: Instituto Tecnológico Metropolitano.

CERTO, Samuel: 2001. Administración Moderna. Bogotá: Prentice Hall.

COSTA-FILHO, A: 2005. Educación Superior y Transformación Productiva. Cab /Convenio Andrés Bello Colciencias - Secretaría Técnica. Oncyt Organismos Nacionales de C\&T del Convenio Andrés Bello.

ECHEVERRÍA, Javier: 2001. Ciencia, tecnología y valores. Hacia un análisis axiológico de la actividad tecnocientífica. En: IBARRA, A. y LÓPEZ, J. A.: 2001, Desafíos y tensiones actuales en ciencia, tecnología y sociedad. Madrid: Biblioteca Nueva OEI. 2002, Ciencia y Valores. Barcelona: Destino.

GUERRERO, Omar: 1997. Principios de administración pública. En la red, http://omega.ilce.edu.mx:3000/sites/csa/principio/leccion1.html\#publico

HABERMAS, Jürgen: 1990. 'Acerca del uso ético, pragmático y moral de la razón práctica'. En: Filosofia, No. 1, Mérida, Venezuela. 1984. Ciencia y técnica como "ideología". Madrid: Tecnos, 2001.

IBARRA, Andoni: 2006. Tecnología para el desarrollo en los países menos desarrollados. Medellín: conferencia en el Instituto Tecnológico Metropolitano, marzo $1^{\circ}$ de 2006.

JARAMILLO, Hernán y CHAPARRO, Fernando: 2004. Evaluación del Impacto del Sistema Nacional de Ciencia y Tecnología: Una primera aproximación. Bogotá: COLCIENCIAS, Universidad del Rosario.

MALDONADO, Carlos Eduardo: 2005. Ciencia y tecnología como políticas públicas y sociales. Bogotá: Universidad Externado de Colombia, Observatorio Colombiano de Ciencia y Tecnología.

MEDELLÍN TORRES, Pedro: 2005. El camino a lo posible: Aportes a la prospectiva científica y tecnológica, desde un enfoque de gobierno y políticas públicas. Bogotá: Fundación Ortega y Gasset, mayo de 2005.

MORIN, Edgar: 1981. Para salir del siglo XX. Barcelona: Kairós.

OLIVÉ, León: 2000. El bien, el mal y la razón: facetas de la ciencia y de la tecnología. México: Paidós-UNAM.-2004a. Normatividad y valores en la ciencia y la tecnología. Simposio: "La ciencia y cómo verla". XV Congreso Iberoamericano de Filosofia. Lima, enero 12-16 de 2004. 
http://www.pucp.edu.pe/eventos/congresos/filosofia/programa_general/martes/sesion 16.45-18.15/OliveLeon.pdf - 2004b. La democratización de la ciencia desde la perspectiva de la ética. México: UNAM, en la red: http:// www.istas.coo.es/escorial04/material/dc15.pdf

PÉREZ RANSANZ, Ana Rosa: 1999. Kuhn y el cambio científico. México: Fondo de Cultura Económica.

QUINTANILLA, Miguel Ángel: 2002. 'La democracia tecnológica'. En: AIBAR, E. y QUINTANILLA, M. Á.: (2002). Cultura tecnológica: estudios de ciencia, tecnología y sociedad. Barcelona: ICE / Horsori Editorial.

RODRÍGUEZ MEDINA, Leandro: 2005. Las políticas públicas como intervención en la realidad. Sistema de Información IIG. En la red: www.iigov. org/biblioteca/readResourece. $d r t ? \mathrm{id}=150$

ROTH DEUBEL, André Noel.: 2004, Políticas Públicas: Formulación, implementación y evaluación. Bogotá: Ediciones Aurora.

SHRUM, Wesley y SHENHAV, Y.: 1995, Science and Technology in Less Developed Countries. P. 627-651. Handbook of science and technology studies. / Editors, Sheila Jasanoff... [et al.] Thousand Oaks: Sage Publications.

VANNEVAR, B.: 1945. La ciencia, la frontera sin fin: un informe al presidente. En: Redes 14, Revista de Estudios Sociales de la Ciencia. Buenos Aires: Universidad Nacional de Quilmas, (1999). En la red: www.oei. es/ctsiima/VANNEVARBUSH 